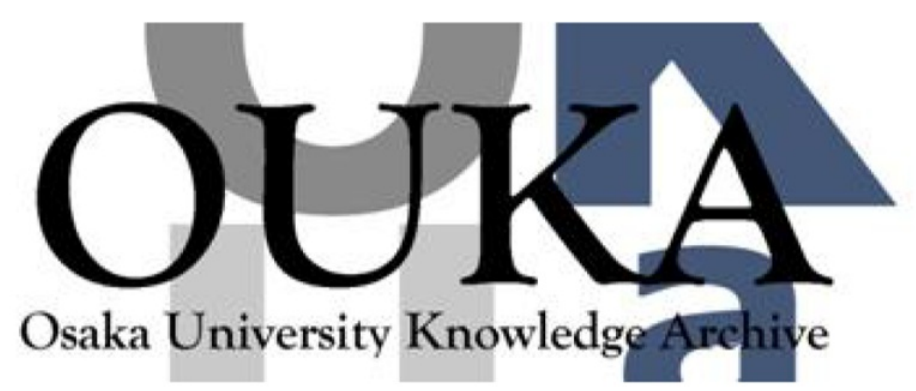

\begin{tabular}{|c|c|}
\hline Title & $\begin{array}{l}\text { Demonstration of bulk acceleration of ions in } \\
\text { ultraintense laser interactions with low- } \\
\text { density foams }\end{array}$ \\
\hline Author (s) & Li, Y. T. ; Sheng, Z. M. ; Ma, Y. Y. et al. \\
\hline Citation & Physical Review E. 72(6) p. 066404 \\
\hline Issue Date & $2005-12$ \\
\hline oaire:version & VoR \\
\hline URL & https://hdl. handle. net/11094/3448 \\
\hline rights & $\begin{array}{l}\text { Li, Y. T., Sheng, Z. M., Ma, Y. Y., Jin, Z., } \\
\text { Zhang, J., Chen, Z. L., Kodama, R, Matsuoka, } \\
\text { T., Tampo, M. 'Tanaka, K. A. ' Tsutsumi, T., } \\
\text { Yabuuchi, T., Du, K., Zhang, H. Q., Zhang, L., } \\
\text { Tang, Y.' J.', Physical Review E, 72, 6, 066404, } \\
\text { 2005-12. "Copyright } 2005 \text { by the American } \\
\text { Physical Society." }\end{array}$ \\
\hline Note & \\
\hline
\end{tabular}

Osaka University Knowledge Archive : OUKA

https://ir. Library. osaka-u. ac. jp/

Osaka University 


\title{
Demonstration of bulk acceleration of ions in ultraintense laser interactions with low-density foams
}

\author{
Y. T. Li, Z. M. Sheng, Y. Y. Ma, Z. Jin, and J. Zhang* \\ Laboratory of Optical Physics, Institute of Physics, Chinese Academy of Sciences, Beijing 100080, People's Republic of China \\ Z. L. Chen, R. Kodama, T. Matsuoka, M. Tampo, K. A. Tanaka, T. Tsutsumi, and T. Yabuuchi \\ Institute of Laser Engineering, Osaka University, 2-6 Yamada-oka, Suita, Osaka 565-0871, Japan \\ K. Du, H. Q. Zhang, L. Zhang, and Y. J. Tang \\ Research Center for Laser Fusion, Mianyang 621900, People's Republic of China \\ (Received 7 July 2004; revised manuscript received 22 February 2005; published 12 December 2005)
}

\begin{abstract}
Ion acceleration inside low-density foams irradiated by ultraintense laser pulses has been studied experimentally and theoretically. It is found that the ion generation is closely correlated with the suppressed hot electron transport inside the foams. Particle-in-cell simulations suggest that localized electrostatic fields with multi peaks around the surfaces of lamellar layers inside the foams are induced. These fields inhibit hot electron transport and meanwhile accelerate ions inside the foams, forming a bulk acceleration in contrast to the surface acceleration at the front and rear sides of a thin solid target.
\end{abstract}

DOI: 10.1103/PhysRevE.72.066404

PACS number(s): 52.38.Kd, 52.50.Jm

\section{INTRODUCTION}

Ion acceleration in high-intensity laser interaction with solid targets has been studied extensively in recent years $[1,2]$. Ions cannot be accelerated directly by laser fields at intensities currently available due to their heavy mass. Typically the laser energy is deposited first into hot electrons [3]. Then part of the electron energy is transferred to the ions through the induced electrostatic field. In the intense-laser interaction with solid foils, usually there are three groups of accelerated ions. The first two occur at the front surface, moving backward and forward, respectively [1], and the third one occurs at the rear surface, moving forward, due to the hot electron transport through the target rear surface [2]. A conducting target usually produces ions at the rear target surface with higher energies than a dielectric target since hot electrons can transport more easily through conductors, where the return currents of free electrons are induced sufficiently to compensate the hot electron current. Since no strong electrostatic field is established inside a solid target, there is no obvious ion acceleration process inside the target bulk. Ion acceleration can be considered as a kind of surface acceleration, occurring both at the rear-side surface and the front-side (or critical density) surface.

In this paper, we demonstrate that, in addition to the surface ion acceleration, ions inside a low-density foam target can also be accelerated to moderate energies. This is referred to as bulk acceleration here to distinguish it from the surface acceleration with a solid target. When hot electrons produced in the ultraintense laser-plasma interactions transport in the foam, strong charge separation fields will be induced around each lamellar layer of the foam in a way similar to laser

\footnotetext{
*Author to whom correspondence should be addressed. Email address: jzhang@aphy.iphy.ac.cn
}

interaction with a single solid foil. Such electrostatic fields result in serious inhibition of the transport of hot electrons and in the meanwhile accelerate ions inside. Comparing with surface acceleration, the number of accelerated ions could be significantly enhanced in the bulk acceleration inside the foam, even though the maximum ion energy is reduced.

\section{EXPERIMENTS}

The experiments were carried out using the PW laser facility Gekko XII and the laser system Gekko Modulate II (GMII) at the Institute of Laser Engineering, Osaka University. The PW laser produces a linearly polarized laser pulse with an energy up to $500 \mathrm{~J}$ and a duration of $0.5-1 \mathrm{ps}$ at the wavelength $1.053 \mu \mathrm{m}$. About $30 \%$ of the laser energy was focused into a $30-\mu \mathrm{m}$ focal spot using an $f / 7$ off-axis parabolic mirror. The contrast ratio was better than $10^{-8}$. The $p$-polarized laser pulses were incident on the target at an angle $26^{\circ}$ to the target normal. In the GMII experiments, 0.6 ps, $1.053 \mathrm{~mm} p$-polarized laser pulses with an energy up to $10 \mathrm{~J}$ were focused by an $f / 3.8$ off-axis parabolic mirror onto the target. The incidence angle of the laser beam was $20^{\circ}$. The diameter of the focus on the target was also about $30 \mu \mathrm{m}$. A prepulse with an energy about $3 \times 10^{-3}$ of the main pulse, originating from the regenerative amplifier, starts from $700 \mathrm{ps}$ ahead of the main pulse peak. Therefore the main laser pulse interacted with a preplasma for all shots in the GMII experiments.

Low-density deuterated foams and solids were used as targets. The target material was deuterated Polystyrene $\left[\left(\mathrm{C}_{2} \mathrm{D}_{3}\right)_{n}\right.$, with $\mathrm{D}$ enrichment greater than $\left.98 \%\right]$. The foams were prepared by the sol-gel-aerosol method. Two different foams with an average density $50 \mathrm{mg} / \mathrm{cm}^{3}$ and $160 \mathrm{mg} / \mathrm{cm}^{3}$, respectively, were used.

The energetic ions inside the bulk targets were diagnosed by neutron spectroscopy produced from nuclear fusion reac- 
tions of $D-D$ collisions [4]. The neutron spectra were measured by single-channel time-of-flight neutron spectrometers and multichannel detector array spectrometers (Mandala) [5]. The energy resolutions were 30 and $100 \mathrm{keV}$ for the Mandala and the single-channel spectrometers, respectively. The neutron spectrometers were used at different viewing angles in the experiments. The accelerated ion momentum distributions inside the target were deduced by fitting the neutron spectra with a three-dimensional (3D) Monte Carlo (MC) code.

The forward ions and hot electrons ejected from the rear target surface were measured by a $40 \times 40$-mm stack of several layers of radiochromic films (RCF), CR39 plastic nuclear track detectors, and imaging plates (IP). The stacks were set parallel to the target surface at a distance $5 \mathrm{~cm}$ from the target. A magnetic spectrometer with $2500-\mathrm{G}$ permanent magnets was aligned in the laser propagation direction to measure the electron energy distribution. The acceptance angle of the spectrometer was $10^{-5} \mathrm{rad}$. The electron energy spectrum was recorded by calibrated imaging plates [6].

\section{RESULTS AND DISCUSSIONS}

\section{A. Neutron spectra and ion momentum distributions inside solid and foam targets}

Neutron emission has been widely studied in ultraintense laser interactions with deuterated solid targets [7]. In most experiments, the neutrons are produced by the collisions of the forward-moving deuterons generated at the front surface with the target atoms, i.e., a beam-target type interaction. The collisions between deuterons can trigger the fusion reaction $d(d, n)^{3} \mathrm{He}$. The energy of the neutrons is $2.45 \mathrm{MeV}$, which is fixed in the center-of-mass frame. However, in the laboratory frame, the measured neutron energy will be shifted due to the Doppler effect. The shift of the peak of the neutron spectra depends on the original energy and direction of deuterons as well as the measurement direction. For example, the shift will be positive if the neutron spectra are measured by a detector facing the movement direction of the deuteron ions, while the shift will be negative if the deuteron ions move away from the detector. In our experiments we measured the neutron emission from a 0.25 -mm-thick CD solid target first. Figure 1 shows the neutron spectrum detected by the Mandala detector at $76^{\circ}$ with respect to the front target normal and at a distance of $13.5 \mathrm{~m}$. The inset illustrates the mutual orientation of the laser beam, the target, and the neutron detector. The laser energy was $160 \mathrm{~J}$. As mentioned in Sec. I, and shown by particle-in-cell (PIC) simulations [see Fig. 5(b)], there are three ion groups produced in the intense laser-solid interactions. Sine the observed neutron signals originate from the nuclear reactions inside the solid, the backward ions produced at the front surface and the forward ions produced at the rear surface do not contribute to the spectra. Only the group of ions moving into the target from the front surface contributes to the nuclear reactions. Therefore the neutron spectra only contain information on the forward-moving deuterons generated at the front target surface. The shift of the spectrum peak from 2.45 MeV to the lower energy indicates that the deuterons

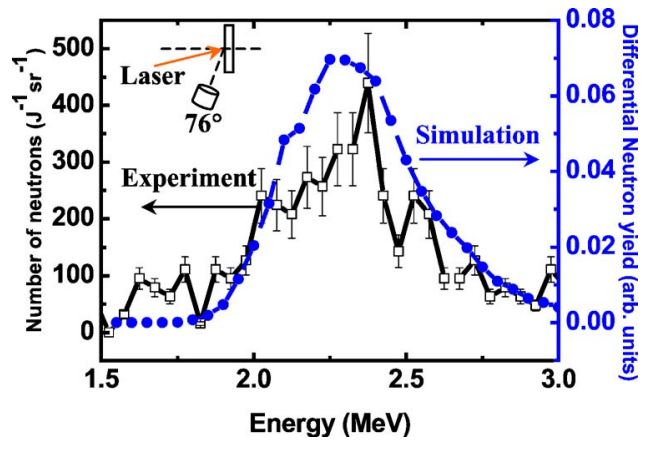

FIG. 1. (Color online) Typical neutron spectrum for a 0.25-mmthick CD solid target, measured at $76^{\circ}$ with respect to the front target normal. The solid and dashed lines show the energy spectra obtained in the experiment and by fitting with Monte Carlo simulations, respectively. The inset illustrates the geometry of the laser beam, the target, and the direction of neutron measurements.

are accelerated forward into the target. This ion group is accelerated by the electric field induced by the longitudinal ponderomotive force of the laser pulse. To deduce the behaviors of the deuterons we have modified the neutron source reaction code, DROSG-2000 [8], to be a 3D MC code, which is appropriate to simulate the nuclear reactions by the large number of deuterons accelerated in the laser-plasma interactions. In all $\mathrm{MC}$ simulations the energy resolution is set to be $50 \mathrm{keV}$. After trying numerous calculations with different ion momentum distributions and angular distributions, we find the experimental neutron spectrum can be fitted well by a simulated one assuming that the deuteron ions are injected into the target along the target normal with a divergent angle $30^{\circ}-40^{\circ}$ (full width at half maximum of a Gaussian distribution) and conform to a Maxwellian energy distribution with a temperature $300-350 \mathrm{keV}$. The simulated spectrum is also shown by the dashed line in Fig. 1. In experiments, the direction of the ion beam depends on the local spatial shape of the critical surface, where the acceleration electric field is induced by the laser ponderomotive force. The distorted critical surface caused by the finite laser diameter leads to divergent emission of the ion beam.

The solid lines in Figs. 2(a) and 2(b) show the experimental neutron spectra for a $1.6-\mathrm{mm}$-thick, $160 \mathrm{mg} / \mathrm{cc}$ foam, measured at $76^{\circ}$ (a) and $16^{\circ}$ (b) to the front target normal, respectively. The laser energy was $103 \mathrm{~J}$. By use of the MC code to fit the neutron spectra, we find that the deuteron ions consist of two components. The dominant component is isotropic and conforms to a Maxwellian energy distribution with a temperature 30-40 keV. Another component, also with the similar temperature $30-40 \mathrm{keV}$, however, is distributed predominantly in the plane parallel to the target surface. It can be attributed partially to hole-boring or shock wave at the target front, which results in radial ion acceleration [9]. Under the present experimental condition, the fraction of the isotropic one to the total ion emission is over $80 \%$. The deduced ion temperatures and angular distributions agree qualitatively with our two-dimensional (2D) particle-in-cell (PIC) simulations as shown in the subsection "simulations."

Figure 3 shows the neutron spectra for a 1-mm-thick, $50-\mathrm{mg} / \mathrm{cc}$ foam, measured at $16^{\circ}$ (a) and $122^{\circ}$ (b) to the front 


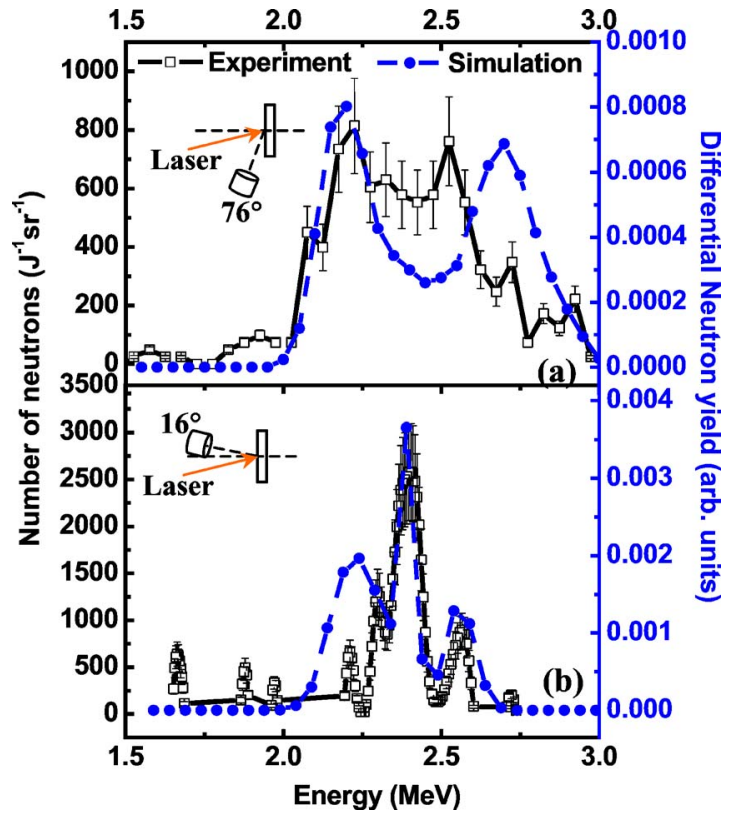

FIG. 2. (Color online) Neutron spectra for a 1.6-mm-thick, $160-\mathrm{mg} / \mathrm{cc} \mathrm{CD}$ foam target, measured at $76^{\circ}$ (a) and $16^{\circ}$ (b) with respect to the front target normal. The solid and dashed lines show the spectra found in the experiment and by fitting with Monte Carlo simulations, respectively.

target normal, respectively. The distance from the detector to the focus was 4.5 and $2.65 \mathrm{~m}$, respectively. The laser energy was $190 \mathrm{~J}$. The deduced ion distribution is also composed of two components, a dominant and isotropic one with a higher temperature 50-70 keV than that of the $160 \mathrm{mg} / \mathrm{cc}$ foam and another that is distributed predominantly in the plane parallel

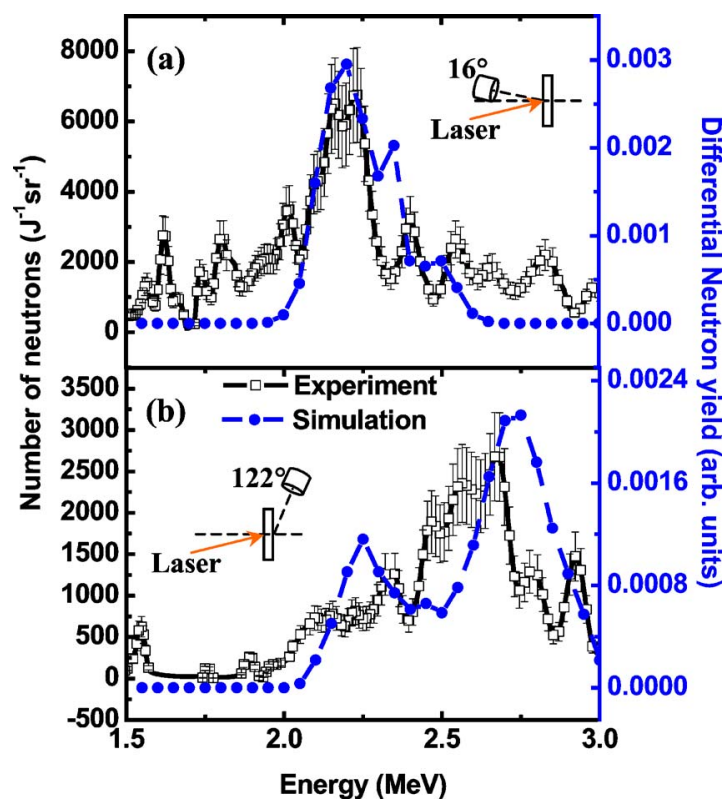

FIG. 3. (Color online) Neutron spectra for a 1-mm-thick, $50-\mathrm{mg} / \mathrm{cc} \mathrm{CD}$ foam target, measured at $16^{\circ}$ (a) and $122^{\circ}$ (b) with respect to the front target normal. The solid and dashed lines show the spectra found in the experiment and by fitting with Monte Carlo simulations, respectively. to the target surface with a temperature $30-40 \mathrm{keV}$. The fraction of the isotropic component is about $90 \%$. There is a large positive energy shift observed behind the target in Fig. $3(\mathrm{~b})$. The detector at $122^{\circ}$ will see a part of the deuterons approaching it, while the other part is moving far away from it. This will lead to two neutron peaks. The peak with the positive shift is higher than the negative one because the reaction probability is higher for small collision angles.

Based on the neutron spectra and MC simulations, one concludes that the deuteron ions are accelerated predominantly in the longitudinal direction with certain divergence angles in the laser solid interaction. While in the laser-foam interaction, accelerated ions are distributed mainly isotropically in all directions. Based on the MC simulations, we estimate the number of the accelerated deuterons to be $0.6-1 \times 10^{13}, 2-6 \times 10^{14}$, and $1-3 \times 10^{15}$ for the solid target, $160-\mathrm{mg} / \mathrm{cc}$ and $50-\mathrm{mg} / \mathrm{cc}$ foam targets, respectively. This suggests that the number of accelerated deuterons for the foam targets is usually larger than for the solid targets. Both the different angular distributions and numbers of ions for different targets indicate that different acceleration mechanisms are involved. In the laser-solid interaction, the acceleration is found mainly at the front and rear surfaces, while in the laser-foam interaction, bulk acceleration can be found inside the targets, as indicated in our simulations. The detailed discussions will be presented in the following subsections.

\section{B. Forward hot electrons ejected from targets}

To understand the ion acceleration inside the foam targets, which is closely correlated with the electron transport inside the targets, the forward hot electrons were also measured. Figures 4(a) and 4(b) show the spatial distributions of the hot electrons with different energy ranges for the solid and $50-\mathrm{mg} / \mathrm{cc}$ foam targets, respectively, measured behind the targets in the GMII laser experiments. The laser energies on the targets were 10.7 and $10.3 \mathrm{~J}$, respectively. The electron beam patterns for both the solid and foam targets appear to be broken up. This is found more obviously for the foam case, where the whole electron beam is even split into two parts in addition to the embodied fine filaments. Note that the filamentation of hot electron beams inside a foam plasma has also been observed very recently [10]. More interestingly, after integrating the counts over the whole area of each imaging plate layer (each layer corresponds to a different energy range), we find that the total signal counts for the foam are much lower than that for the solid. This suggests that the transport of hot electrons is seriously suppressed inside the foams. This suppression can also be seen in Fig. 4(c), which shows typical electron spectra for the 0.25 -mm-thick solid and 1.6-mm-thick, $160-\mathrm{mg} / \mathrm{cc}$ foam target.

The inhibition of the hot electron transport in foams can be explained by the following scenario. The current of hot electron beam produced in the experiments is greater than the Alfvén limit, the maximum current that can be propagated by an electron beam in vacuum [11]. For currents exceeding the limit, the intense self-consistent magnetic field associated with the beam will deflect the electron trajectories 

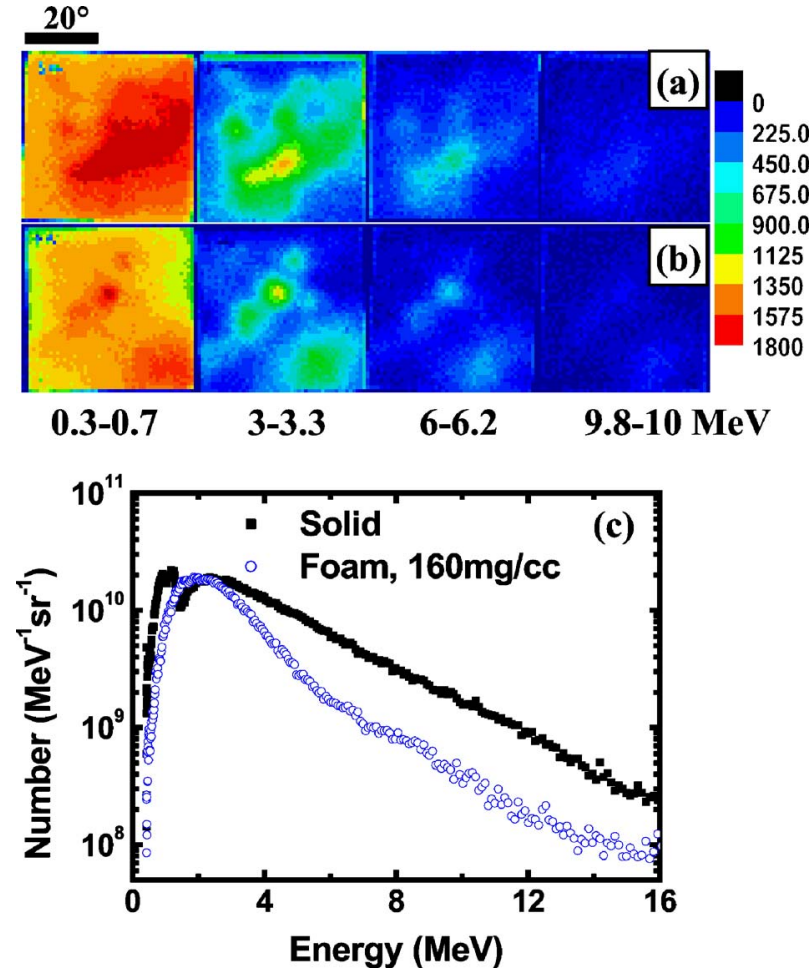

FIG. 4. (Color online) Spatial distributions of hot electrons for a 0.25-mm-thick solid (a), and a 0.25-mm-thick, 50-mg/cc foam (b), as well as typical energy spectra for $0.25 \mathrm{~mm}$ solid and $1.7 \mathrm{~mm}$, $160-\mathrm{mg} / \mathrm{cc}$ foam (c) obtained in the GMII laser experiments.

back and prevent their forward movement. However, such a huge hot electron current can propagate in solid targets because the background cold electron current can neutralize the space charge of the beam and slow down the hot electrons. Therefore the hot electron transport strongly depends on the conductivity [12] and the density of the target material. Studies have shown that the transport in dielectric targets [13] and low density gas targets $[14,15]$ is inhibited more significantly than that in metal targets. For foam targets, on one hand the return current is weaker than that in solids since there are fewer cold background electrons due to their low average density. On the other hand, there will be multipeak electric fields induced around the lamellar layers inside the foams when hot electron transport to the region (see the section of simulations). As a result, a significant inhibition of the hot electron beam is generated inside the foam, as observed in the experiments. The similar inhibition of hot electrons in plastic foams has also been observed by $K \alpha$ spectroscopy [16].

\section{Forward ions ejected from targets}

The measurement of the ejected ions provides another diagnostic for the hot electron transport and ion acceleration. The ion signals measured behind a target, if detectable, can consist of three parts: two originate from the front and rear surfaces, respectively, and the third one from the inside of the target bulk. Ions from the front surface and bulk targets have to be energetic enough to pass through the targets, while those from the rear surface, even generated at moderate energy, can be detected easily. For the foam targets, ion signals were detectable only for the foams with a thickness less than $0.25 \mathrm{~mm}$ with the GMII laser pulses. For the foam targets thicker than $1 \mathrm{~mm}$, even irradiated by the PW laser pulses in Gekko XII laser, no ion signal was recorded. However, for the solid targets with much larger areal density than these foams, say, $0.25-\mathrm{mm}$-thick solid, there were still strong ion signals on the stack. By changing the filter thickness in ion measurements, it is found that for a $50-\mathrm{mg} / \mathrm{cc}, 0.25-\mathrm{mm}$ thick foam target the maximum energy of deuterons measured is estimated to be within $1-2 \mathrm{MeV}$; while for a 0.25 mm-thick solid target the maximum energy of protons originating from the rear surface is within 5-7 MeV, which agrees with the simulation results at the similar intensity as shown later [see Fig. 6(d)].

No ion signal detected behind the thicker foams indicates that the ions do not originate from the rear surface, because if the ions were from the rear surface they should be detectable on the detector no matter how thick the target is. Taking into account the reduction of hot electron signals discussed above, the missing of ion signals with the thick foam targets can be interpreted by the great reduction of the number and energy of electrons reaching the rear side of the targets because of the significant transport inhibition of hot electrons inside the foam targets. Thus a sheath electrostatic field cannot be built up sufficiently to accelerate ions at the rear surface.

\section{Simulations}

In order to further clarify the physical processes associated with ion acceleration in the foams, one-dimensional (1D) and 2D fully relativistic PIC simulations have been conducted. In the 2D simulations, a $p$-polarized laser pulse with a wavelength of $1.053 \mu \mathrm{m}$ is incident at $20^{\circ}$ onto the target with an intensity of $2.0 \times 10^{19} \mathrm{~W} / \mathrm{cm}^{2}$. The diameter of the laser focus is $10 \mu \mathrm{m}$. To model the laser interaction with a foam target, we use an initial electron density profile shown in Fig. 5(a), which is composed of an underdense plasma region plus a high-density region. The electron density in the underdense plasma increases from 0 to $1 n_{c}$ linearly in a scale length of $20 \lambda_{0}$ to imitate the preplasma in our experiments, where $n_{c}$ is critical density and $\lambda_{0}$ is laser wavelength, respectively. In the high-density region the plasma density is distributed to square grid borderlines to imitate the real structure in a foam target, which is composed of separated lamellar layers distributed randomly. Even though our model adopts a simplified regular distribution for the lamellar structure, the involved physical process with ion acceleration is qualitatively the same for the two cases. The thickness of the grid borderlines is $0.1 \lambda_{0}$, the grid size is $1 \lambda_{0} \times 1 \lambda_{0}$, and the density on the grid borderlines is $9 n_{c}$. The total numbers of electrons and ions in the simulation box are around 6000000 each. For the laser-solid interaction, the region with grid structures is substituted with a single overdense plasma block in a thickness of $8 \lambda_{0}$.

Figures 5(b) and 5(c) show the longitudinal and transverse momenta of deuteron ions in the phase space $\left(p_{x}, x\right)$ and 

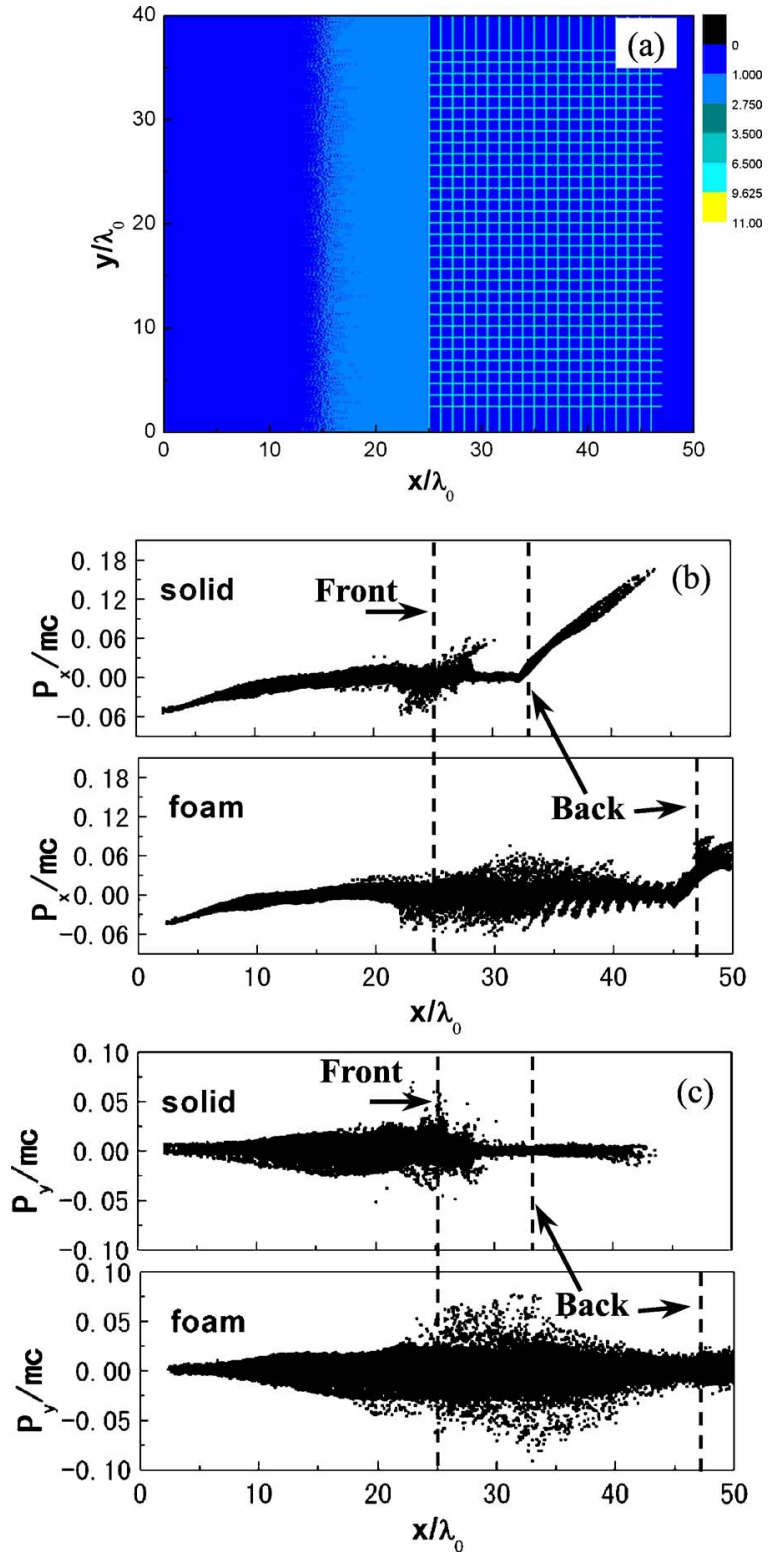

FIG. 5. (Color online) Initial electron density distribution used in the 2D PIC simulations (a); longitudinal (b) and transverse (c) momenta at $t=150$ laser cycles both for solid and foam targets, respectively. The dash lines in (b) and (c) indicate the initial front and rear surfaces of the targets.

$\left(p_{y}, x\right)$ for both the solid and foam targets at $t=150$ laser cycles, respectively. For the solid target, deuterons both at the front and rear surfaces are accelerated. The ions at the rear surface are accelerated only in the longitudinal direction. However, the ions injected to the target at the front surface move in both longitudinal and transverse directions. This can lead to a divergent ion beam into the target bulk, as indicated by the neutron spectra. Once they propagate away from the front surface, the acceleration terminates. The maximum energy of ions from the rear surface is higher than that from the front surface. Ions initially located inside the solid do not gain energy since there is almost no electrostatic field induced inside because of the presence of compensating return currents. For the foam target, however, the deuterons inside the target are also accelerated in addition to those at the front and rear surfaces. Moreover, the ions inside the foam are accelerated in both forward and backward directions in the longitudinal space $\left(p_{x}, x\right)$, as well as in the transverse space $\left(p_{y}, x\right)$. The energy of the ions is moderate compared with the ions originated at the rear surface of the solid target, and is comparable to (or even larger for a thicker target than) those at the rear surface of the foam target. The number of ions accelerated inside the bulk foam is much larger than that accelerated at the rear surface of a solid target. Thus the ion acceleration inside the target contributes to the dominant isotropic component deduced from the MC simulations. In addition, one can also see that the maximum transverse momentum $p_{y}$ is slightly larger than the longitudinal momentum $p_{x}$. This implies that a few ions are accelerated more energetically transversely, which may be related with the presence of the secondary transverse component of ions deduced from the neutron spectra by the MC simulations.

Our 1D simulation results illustrate even more clearly how the ions inside the foam are accelerated. In the simulations, the electron density in the underdense plasma increases from $0.01 n_{c}$ to $n_{c}$ exponentially in a scale length of $40 \lambda_{0}$ [see Fig. 6(a)]. The high-density region consists of 40 thin layers of overdense plasma at a density of $9 n_{c}$ in the $x$ direction to imitate the foam structure. The thickness of each layer is $0.1 \lambda_{0}$ and the separation between neighboring layers is $\lambda_{0}$, as similar with the 2D simulation parameters along the $x$ direction. Although this is much simplified from the real foam structure, such a model can be helpful to reveal the main physical processes involved along a given direction. The target consists of fully ionized deuterons (ion mass is $3680 m_{e}$ ), $\mathrm{D}^{+}$, and carbon ions with ionization of $4, \mathrm{C}^{+4}$. The incident laser intensity is $4.0 \times 10^{18} \mathrm{~W} / \mathrm{cm}^{2}$. Figure $6(\mathrm{~b})$ shows the induced longitudinal electric fields for the foam and solid targets, as well as the electron density for the foam target at $t=400$ laser cycles. In the case with the solid foil, when the hot electrons produced in the laser-plasma interaction transport through the rear target surface, an electrostatic field is built up behind, which decays with the distance from the rear surface. This field accelerates ions from the rear surface into the vacuum as shown in Fig. 5. This is the so-called target normal sheath acceleration (TNSA). However, in the case with the foam target, an electrostatic field with multi peaks is built up around each lamellar layer inside the bulk foam when hot electrons transport inside it, as shown in Fig. 6(b). It is this multipeaked field that accelerates the ions inside the foam. Figure 6(c) shows the deuteron density inside the foam target at $t=300$ and 500 as well as the electric field at $t=500$. At $t=300$, most of the deuterons are still located at their initial positions. However, at $t=500$, most of them have been pushed away from their "parent" layers. Note that the electric field induced around each lamellar layer appears to be ambipolar, so that deuterons at the two sides are accelerated in opposite directions, as found in the $2 \mathrm{D}$ simulations [see Fig. 5(b)]. This ambipolar field structure is partially caused by the return electron current around individual overdense lamellar layers.

Figure 6(d) shows the energy distribution of deuteron ions at $t=500$ laser cycles for both the solid and foam targets. It demonstrates that more deuterons with moderate energies are 

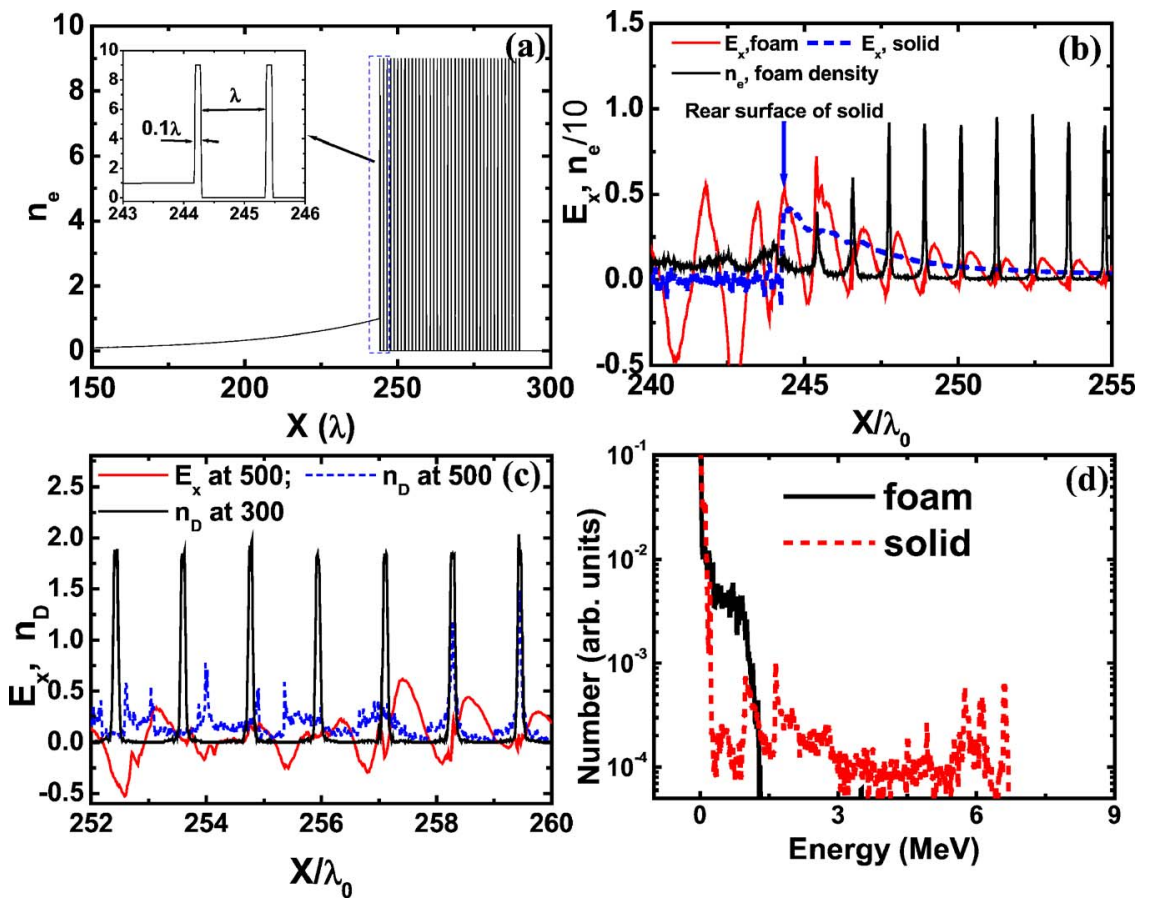

produced in the foam target than in the solid target due to the acceleration inside the foam.

Therefore both our 1D and 2D simulations suggest that there is a bulk acceleration process inside a foam target due to the multi peaked charge separation fields induced around the lamellar layers. From the simulations, it is noted that ions accelerated inside bulk foams are less energetic than that produced at the rear surface of the solid targets. This can be attributed to two reasons. One is that the acceleration length inside foams is only limited to a distance between adjacent overdense layers, which is smaller than that behind the solid rear surface. Another is that the local electric field around each overdense lamellar layer evolves into an ambipolar structure at a later stage as mentioned before [see Fig. 6(c)]. The ions will encounter a deceleration field when they escape far from the "parent" layers and move close to adjacent layers. Therefore increasing the distance between adjacent layers (or decreasing the foam density) can lead to larger ion energy. This has been verified by our simulations. This can also explain that the temperature and the number of ions produced in the $50-\mathrm{mg} / \mathrm{cc}$ foam are larger than that in the $160-\mathrm{mg} / \mathrm{cc}$ foam. One expects that there exists an optimized foam density for bulk acceleration for given laser parameters.

From the viewpoint of neutron generation, there is a significant difference between the solid and foam targets. For the solid case, only the deuterons produced at the front surface and moving into the target contribute to neutron generation by colliding with the background cold deuterons inside the target. However, for the foam case the energetic ions produced inside the foam due to the bulk acceleration will contribute directly to the nuclear reactions. Because the lamellar layers are distributed randomly in a real foam target, the ions from bulk acceleration tend to be predominantly isotropic in momentum space, as shown by the deduced ion distributions from the neutron spectra.
FIG. 6. (Color online) (a) Initial electron density distribution used in the 1D PIC simulations. (b) Electric fields inside the foam (gray solid line) and the fields behind the rear surface of solid target (dashed line) at $t=400$ laser cycles. The electron density (divided by a factor of 10) at the same time is also shown (black solid line). (c) The deuteron densities inside the foam target at $t$ $=300$ (black solid line), $t=500$ (dashed line), and the longitudinal electric field at $t=500$ (gray solid line). (d) Energy distributions of deuteron ions at $t=500$ both for solid and foam targets.

\section{SUMMARY}

When hot electrons produced in the ultraintense laserplasma interactions transport in a low-density foam target, they are greatly suppressed due to the low-average density and the density discontinuities inside the target. Thus only a smaller number of electrons can reach the target rear side to create target normal sheath ion acceleration. Most of the electron-beam energy is transferred to the deuterons inside the foam through the electric fields induced by the unique structure of the foam. As a result, significant ion acceleration occurs inside the foam, forming the bulk acceleration. The bulk acceleration is a different scenario from the simple surface acceleration in the following aspects: (i) the energetic ions are mainly generated inside the foam target; (ii) the acceleration direction of the ions inside the foam is predominantly isotropic, different from the forward acceleration for the solid case; (iii) the acceleration field exhibits multi peaks inside a foam target, instead of decreasing monotonically with distance behind of a solid target; (iv) the ion energy from the inner layers of a foam is moderate as compared with that from the rear surface of a solid; (v) the number of accelerated ions in a foam target can be much larger than that in a solid target. Our results show that a foam or multilayered target could potentially be an efficient means for generating a huge ion flux at moderate energies as well as a table-top neutron source.

\section{ACKNOWLEDGMENTS}

This work was supported by the NNSFC (Grant Nos. 10374115, 60321003, 10335020, 10425416, 10390161, and 10505031, the National High-Tech ICF program, National Key Laboratory of High Temperature and High Density Plasma, and the JSPS-CAS Core University Program on Plasma Physics and Nuclear Fusion. 
[1] E. L. Clark, K. Krushelnick, J. R. Davies, M. Zepf, M. Tatarakis, F. N. Beg, A. Machacek, P. A. Norreys, M. I. K. Santala, I. Watts, and A. E. Dangor, Phys. Rev. Lett. 84, 670 (2000); M. Zepf, E. L. Clark, F. N. Beg, R. J. Clarke, A. E. Dangor, A. Gopal, K. Krushelnick, P. A. Norreys, M. Tatarakis, U. Wagner, and M. S. Wei, ibid. 90, 064801 (2003); M. Kaluza, J. Schreiber, M. I. K. Santala, G. D. Tsakiris, K. Eidmann, J. Meyer-ter-Vehn, and K. J. Witte, ibid. 93, 045003 (2004); M. S. Wei, S. P. D. Mangles, Z. Najmudin, B. Walton, A. Gopal, M. Tatarakis, A. E. Dangor, E. L. Clark, R. G. Evans, S. Fritzler, R. J. Clarke, C. Hernandez-Gomez, D. Neely, W. Mori, M. Tzoufras, and K. Krushelnick, ibid. 93, 155003 (2004); A. Macchi, F. Cattani, T. V. Liseykina, and F. Cornolti, ibid. 94, 165003 (2005).

[2] S. Wilks, A. B. Langdon, T. E. Cowan, M. Roth, M. Singh, S. Hatchett, M. H. Key, D. Pennington, A. MacKinnon, and R. A. Snavely, Phys. Plasmas 8, 542 (2001); S. P. Hatchett, C. G. Brown, T. E. Cowan, E. A. Henry, J. S. Johnson, M. H. Key, J. A. Koch, A. B. Langdon, B. F. Lasinski, R. W. Lee, A. J. Mackinnon, D. M. Pennington, M. D. Perry, T. W. Phillips, M. Roth, T. C. Sangster, M. S. Singh, R. A. Snavely, M. A. Stoyer, S. C. Wilks, and K. Yasuike, ibid. 7, 2076 (2000); M. Hegelich, S. Karsch, G. Pretzler, D. Habs, K. Witte, W. Guenther, M. Allen, A. Blasevic, J. Fuchs, J. C. Gauthier, M. Geissel, P. Audebert, T. Cowan, and M. Roth, Phys. Rev. Lett. 89, 085002 (2002); A. Pukhov, ibid. 86, 3562 (2001); M. Roth, A. Blazevic, M. Geissel, T. Schlegel, T. E. Cowan, M. Allen, J.-C. Gauthier, P. Audebert, J. Fuchs, J. Meyer-ter-Vehn, M. Hegelich, S. Karsch, and A. Pukhov, Phys. Rev. ST Accel. Beams 5, 061301 (2002); R. A. Snavely, M. H. Key, S. P. Hatchett, T. E. Cowan, M. Roth, T. W. Phillips, M. A. Stoyer, E. A. Henry, T. C. Sangster, M. S. Singh, S. C. Wilks, A. MacKinnon, A. Offenberger, D. M. Pennington, K. Yasuike, A. B. Langdon, B. F. Lasinski, J. Johnson, M. D. Perry, and E. M. Campbell, Phys. Rev. Lett. 85, 2945 (2000); M. Allen, P. K. Patel, A. Mackinnon, D. Price, S. Wilks, and E. Morse, ibid. 93, 265004 (2004); T. E. Cowan, J. Fuchs, H. Ruhl, A. Kemp, P. Audebert, M. Roth, R. Stephens, I. Barton, A. Blazevic, E. Brambrink, J. Cobble, J. Fernandez, J.-C. Gauthier, M. Geissel, M. Hegelich, J. Kaae, S. Karsch, G. P. Le Sage, S. Letzring, M. Manclossi, S. Meyroneinc, A. Newkirk, H. Pépin, and N. RenardLeGalloudec, ibid. 92, 204801 (2004).

[3] R. Kodama, K. Mima, K. A. Tanaka, Y. Kitagawa, H. Fujita, K. Takahashi, A. Sunahara, K. Fujita, H. Habara, T. Jitsuno, Y. Sentoku, T. Matsushita, T. Miyakoshi, N. Miyanaga, T. Norimatsu, H. Setoguchi, T. Sonomoto, M. Tanpo, Y. Toyama, and T. Yamanaka, Phys. Plasmas 8, 2268 (2001).

[4] H. Habara, R. Kodama, Y. Sentoku, N. Izumi, Y. Kitagawa, K. A. Tanaka, K. Mima, and T. Yamanaka, Phys. Rev. E 69, 036407 (2004); N. Izumi, Y. Sentoku, H. Habara, K. Takahashi, F. Ohtani, T. Sonomoto, R. Kodama, T. Norimatsu, H.
Fujita, Y. Kitagawa, K. Mima, K. A. Tanaka, and T. Yamanaka, Phys. Rev. E 65, 036413 (2002)

[5] N. Izumi, K. Yamaguchi, T. Yamagajo, T. Nakano, T. Kasai, T. Urano, H. Azechi, S. Nakai, and T. Iida, Rev. Sci. Instrum. 70, 1221 (1999).

[6] K. A. Tanakaa, T. Yabuuchi, T. Sato, R. Kodama, Y. Kitagawa, T. Takahashi, T. Ikeda, Y. Honda, and S. Okuda, Rev. Sci. Instrum. 76, 013507 (2005).

[7] P. A. Norreys, A. P. Fews, F. N. Beg, A. R. Bell, A. E. Dangor, P. Lee, M. B. Nelson, H. Schmidt, M. Tatarakis, and M. D. Cable, Plasma Phys. Controlled Fusion 40, 175 (1998); L. Disdier, J-P. Garconnet, G. Malka, and J-L. Miquel, Phys. Rev. Lett. 82, 1454 (1999); D. Hilscher, O. Berndt, M. Enke, U. Jahnke, P. V. Nickles, H. Ruhl, and W. Sandner, Phys. Rev. E 64, 016414 (2001); C. Toupin, E. Lefebvre, and G. Bonnaud, Phys. Plasmas 8, 1011 (2001).

[8] M. Drosg, DROSG-2000, codes and database for 59 neutron source reactions, documented in the IAEA Report No. IAEANDS-87 Rev. 8 (January 2003), received from the IAEA Nuclear Data Section.

[9] A. Pukhov, Z. M. Sheng, and J. Meyer-ter-Vehn, Phys. Plasmas 6, 2847 (1999); G. Pretzler, A. Saemann, A. Pukhov, D. Rudolph, T. Schatz, U. Schramm, P. Thirolf, D. Habs, K. Eidmann, G. D. Tsakiris, J. Meyer-ter-Vehn, and K. J. Witte, Phys. Rev. E 58, 1165 (1998); S. C. Wilks, W. L. Kruer, M. Tabak, and A. B. Langdon, Phys. Rev. Lett. 69, 1383 (1992).

[10] R. Jung, J. Osterholz, K. Löwenbrück, S. Kiselev, G. Pretzler, A. Pukhov, O. Willi, S. Kar, M. Borghesi, W. Nazarov, S. Karsch, R. Clarke, and D. Neely, Phys. Rev. Lett. 94, 195001 (2005).

[11] H. Alfvén, Phys. Rev. 55, 425 (1939).

[12] A. R. Bell, J. R. Davies, S. Guerin, and H. Ruhl, Plasma Phys. Controlled Fusion 39, 653 (1997).

[13] F. Pisani, A. Bernardinello, D. Batani, A. Antonicci, E. Martinolli, M. Koenig, L. Gremillet, F. Amiranoff, S. Baton, J. Davies, T. A. Hall, D. Scott, P. Norreys, A. Djaoui, C. Rousseaux, P. Fews, H. Bandulet, and H. Pepin, Phys. Rev. E 62, R5927 (2000).

[14] D. Batani, S. D. Baton, M. Manclossi, J. J. Santos, F. Amiranoff, M. Koenig, E. Martinolli, A. Antonicci, C. Rousseaux, M. R. Le Gloahec, T. Hall, V. Malka, T. E. Cowan, J. King, R. Freeman, M. Key, and R. Stephens, Phys. Rev. Lett. 94, 055004 (2005).

[15] M. Tatarakis, F. N. Beg, E. L. Clark, A. E. Dangor, R. D. Edwards, R. G. Evans, T. J. Goldsack, K. W. D. Ledingham, P. A. Norreys, M. A. Sinclair, M-S. Wei, M. Zepf, and K. Krushelnick, Phys. Rev. Lett. 90, 175001 (2003).

[16] D. Batani, A. Antonicci, F. Pisani, T. A. Hall, D. Scott, F. Amiranoff, M. Koenig, L. Gremillet, S. Baton, E. Martinolli, C. Rousseaux, and W. Nazarov, Phys. Rev. E 65, 066409 (2002). 